\title{
Discurso socioemocional y construcción compartida del conocimiento en tareas colaborativas en línea
}

\author{
Socioemotional Discourse and Shared Knowledge Construction in Online Collaborative Tasks
}

\section{Discurso sócio-emocional e construção de conhecimento compartilhado em tarefas colaborativas on-line}

Juan Carlos Castellanos-Ramírez

Universidad Autónoma de Baja California

Baja California, México

jcasterra@gmail.com

https://orcid.org/0000-0002-0682-9085

Shamaly Alhelí Niño-Carrasco Universidad Autónoma de Baja California

Baja California, México

shamaly.nino@uabc.edu.mx

iD https://orcid.org/0000-0002-5623-4418

Karla Lariza Parra-Encinas

Universidad Autónoma de Baja California

Baja California, México

parra.karla@uabc.edu.mx

https://orcid.org/0000-0002-2855-2224

Recibido • Received • Recebido: 20 / 06 / 2018

Corregido • Revised • Revisado: 21 / 10 / 2019

Aceptado • Accepted • Aprovado: 11/ 02 / 2020

Resumen: El discurso socioemocional del estudiantado tiene un papel fundamental en el aprendizaje colaborativo en línea, por lo que es necesario que se construya un clima de confianza mutua que permita sostener discusiones profundas, críticas y constructivas sobre los contenidos de la tarea para avanzar en su conocimiento grupal. El objetivo del presente trabajo de investigación fue explorar el discurso socioemocional que los grupos de estudiantes utilizan en distintas fases del proceso de construcción compartida del conocimiento. La investigación se llevó a cabo en una universidad pública mexicana en la que se analizaron procesos colaborativos asíncronos en línea, desarrollados por tres grupos de estudiantes. Mediante la técnica de análisis de contenido se codificaron las contribuciones realizadas por estudiantes en los foros asíncronos de trabajo grupal. Los resultados destacan siete diferentes tipos de discurso socioemocional que los grupos de estudiantes emplean de 
http://doi.org/10.15359/ree.24-2.4

ROR: https://ror.org/01t466c14 Universidad Nacional, Costa Rica

http://www.una.ac.cr/educare

educare@una.cr

manera recurrente durante su colaboración y que inciden de manera importante en la construcción del conocimiento. Se concluye que un discurso socioemocional denso, frecuente y heterogéneo, en cuanto a su tipología, sienta las bases necesarias para que los grupos de estudiantes desarrollen procesos colaborativos exitosos y logren altos niveles de construcción compartida del conocimiento.

Palabras claves: Aprendizaje colaborativo; aprendizaje en línea; comunicación; asíncrona; proceso de aprendizaje; psicología de la educación.

\begin{abstract}
The socioemotional discourse of students plays a fundamental role in online collaborative learning. Therefore, they must build a climate of mutual trust that allows them to hold deep, critical, and constructive discussions about the task's contents, to improve their construction of knowledge. The research was carried out in a Mexican public university where asynchronous collaborative processes developed by three groups of students were analyzed online. The socio-emotional discourse that groups of students use in different phases of the process of shared knowledge construction is explored through the technique of content analysis. The results highlight, on the one hand, seven different types of social-emotional discourse that groups of students frequently use during their collaboration process. On the other hand, it seems that the frequency has an important impact on the construction of knowledge. It is concluded that a dense, frequent, and heterogeneous socio-emotional discourse, in terms of its typology, provides the necessary bases for student groups to develop successful collaborative processes and achieve high levels of shared knowledge construction.
\end{abstract}

Keywords: Collaborative learning; online learning; communication; asynchronous; learning process; educational psychology.

Resumo: O discurso sócio emocional de estudantes tem um papel fundamental na aprendizagem colaborativa on-line, por isso é necessário construir um clima de confiança mútua que permita discussões profundas, críticas e construtivas sobre os conteúdos da tarefa para avançar o conhecimento do grupo. O objetivo desta pesquisa foi explorar o discurso sócio emocional que os grupos de estudantes utilizam em diferentes fases do processo de construção do conhecimento compartilhado. A pesquisa foi realizada em uma universidade pública mexicana, onde foram analisados processos colaborativos assíncronos on-line, desenvolvidos por três grupos de estudantes. Usando a técnica de análise de conteúdo, as contribuições feitas pelos alunos nos fóruns assíncronos de trabalho em grupo foram codificadas. Os resultados destacam sete tipos diferentes de discurso sócio emocional que os grupos de estudantes utilizam de forma recorrente durante sua colaboração e que têm um impacto importante na construção do conhecimento. Conclui-se que um discurso sócio emocional denso, frequente e heterogêneo, em termos de sua tipologia, lança as bases necessárias para que grupos de estudantes desenvolvam processos colaborativos bem-sucedidos e alcancem altos níveis de construção de conhecimento compartilhado.

Palavras chave: Aprendizagem colaborativa; aprendizagem on-line; comunicação; assíncrona; processo de aprendizagem; psicologia educacional. 


\section{Introducción}

El estudio del trabajo colaborativo como método instruccional y los beneficios que esta forma de organización en el aula produce en los aprendizajes del estudiantado tiene una larga tradición investigativa dentro del campo de la psicología educativa (Castellanos Ramírez, et al. 2016; Kyndt et al., 2013; Retnowati et al., 2017). Los primeros trabajos en el ámbito se enfocaron en valorar en qué medida y bajo qué circunstancias las tareas colaborativas producían, en contraste con la realización de tareas educativas individuales o competitivas (Dillenbourg et al., 1996; Johnson y Johnson, 1999), mejores resultados de aprendizaje al desarrollarse procesos interpsicológicos de regulación y construcción compartida del conocimiento (Engel y Onrubia, 2013; Kirschner et al., 2011; Malmberg et al., 2017).

En años recientes ha emergido un fuerte interés por explorar las potencialidades de las TIC como herramientas de apoyo a la colaboración y los efectos que determinados usos de dichas tecnologías producen sobre los aprendizajes del estudiantado (Jeong y Hmelo-Silver, 2016; Kirschner y Erkens, 2013). En este contexto se ha fundado, dentro de las ciencias de la educación, una nueva comunidad de investigación denominada aprendizaje colaborativo mediado por ordenador "Computer-Supported Colaborative Learning, CSCL".

A pesar de las ventajas tecnológicas del CSCL, las investigaciones evidencian que en dichos entornos el estudiantado tiene dificultades para sostener un diálogo productivo sobre los contenidos temáticos de la tarea y, a menudo, no alcanza niveles profundos de construcción compartida del conocimiento (Engel y Onrubia, 2013; Liang et al. , 2010; Rimor et al., 2010; Zhang, 2013); de acuerdo con Johnson (2016), el hecho de que la tecnología permita desarrollar procesos de colaboración en línea, no significa que automáticamente se produzcan interacciones positivas y de provecho para al aprendizaje del estudiantado.

Trabajos previos realizados por Dennen y Wieland (2007), Engel y Onrubia (2013), Rimor et al. (2010) y Zhang (2013) ponen de manifiesto que la colaboración del estudiantado en CSCL muchas veces se limita a la acumulación de ideas o agregados de información simples, sin que ello involucre necesariamente una reflexión profunda sobre la información o ideas aportadas; así mismo se ha observado que cuando el estudiantado acelera la entrega de los productos tiende a establecer consensos superficiales sobre los significados de la tarea sin llevar a cabo un ejercicio argumentativo de las ideas. Al parecer, estos resultados no han sido muy alentadores y se ajustan poco a las expectativas proyectadas por los entes directivos de los centros educativos en torno a los beneficios que el CSCL comporta para los aprendizajes del estudiantado.

En coincidencia con las aportaciones de Bakhtiar et al. (2018), Colomina y Remesal (2015), Järvenoja et al. (en prensa), Kwon et al. (2014) y Zschocke et al. (2016), el aprendizaje colaborativo es un sistema de actividad complejo en el que el estudiantado, además de regular el discurso cognitivo sobre los contenidos temáticos de la tarea, necesita construir un contexto socioemocional 
http://doi.org/10.15359/ree.24-2.4

ROR: https://ror.org/01t466c14 Universidad Nacional, Costa Rica

http://www.una.ac.cr/educare

educare@una.cr

positivo que le permita desarrollar y sostener interacciones productivas. Como destacan Kwon et al. (2014), la interacción socio-emocional es como el aceite de motor que lubrica las actividades entre los miembros de un grupo y que, además, los protege de la fricción al momento de colaborar. Como se ve, las interacciones socioemocionales están estrechamente asociadas con los procesos cognitivos, la formación y el sentimiento de pertinencia a la comunidad.

En este marco, el propósito del presente trabajo consiste en indagar los procesos socioemocionales implicados en el aprendizaje colaborativo en línea. Para ello, mediante la técnica de análisis de contenido se explora el discurso socioemocional que distintos grupos de estudiantes utilizan durante la elaboración de tareas colaborativas en línea, haciendo especial énfasis en el efecto que dicho discurso tiene sobre los procesos de construcción compartida del conocimiento.

\section{Estado de la cuestión}

En los últimos años se ha producido una fuerte cantidad de estudios en torno al CSCL, sin embargo, gran parte de las investigaciones realizadas se han centrado en explorar el aprendizaje y los resultados asociados con el conocimiento de los grupos (Castellanos Ramírez y Onrubia Goñi, 2015; Järvelä y Hadwin 2013), mientras que otros aspectos implicados en el aprendizaje colaborativo, tales como la motivación y la gestión socioemocional, han recibido menor atención por parte de las investigaciones. Tal como destaca Bakhtiar et al. (2018), un aspecto hasta cierto punto descuidado dentro de los diseños instruccionales en aprendizaje colaborativo remite al poco conocimiento empírico que hay con respecto a la importancia y el efecto de la dimensión socioemocional en las interacciones estudiantiles.

Desde el campo de la psicología de la educación, el término socioemocional se utiliza para referirse a los sentimientos, estados de ánimo, afecto y sentido de bienestar de un colectivo (Bakhtiar et al., 2018). Por su parte, la definición operacional del término remite a los intercambios intencionales (habitualmente intercambios de comunicación) entre miembros de un grupo que contribuyen al establecimiento de un clima de confianza mutua entre participantes y que permite el funcionamiento armónico del grupo (Kwon et al. 2014).

Uno de los trabajos pioneros en torno a la dimensión socioemocional del aprendizaje colaborativo en línea fue realizado por Janssen et al. (2012), quienes constataron que un clima socioemocional positivo en los grupos ayuda a que el estudiantado exprese sus desacuerdos más fácilmente y contribuye a la realización de opiniones críticas, divergentes y exploratorias. No obstante, otros trabajos como los de Splichal et al. (2018) y Näykki et al. (2014) destacan que, en entornos de CSCL, establecer un contexto socioemocional positivo entre las personas participantes puede resultar problemático, ya que, a diferencia de la colaboración presencial 
en donde el estudiantado comparte sus emociones o estados motivacionales a través de la comunicación no verbal (expresiones faciales, gestos, lenguaje corporal, contacto táctil y visual), en la colaboración en línea esta información es invisible y requiere un alto grado de explicitación de las emociones, afectos y expresiones de apoyo a través del discurso escrito.

Investigaciones recientes han constatado diferentes tipos de discurso socioemocional que el estudiantado utiliza durante la elaboración de tareas colaborativas. Por ejemplo, Zheng y Huang (2016) estudiaron el discurso afectivo que distintos grupos de estudiantes emplearon durante su colaboración a través del chat; los resultados demostraron que los sentimientos perspicaces (palabras que animan al abordaje de la tarea) afectan positivamente en el rendimiento de los grupos, mientras que el exceso de bromas se vincula con bajos niveles de rendimiento sobre la tarea académica.

De manera similar, Kwon et al. (2014) investigaron tareas colaborativas en línea realizadas por estudiantes de medicina. Los resultados revelaron una relación importante entre la frecuencia del discurso socioemocional utilizado por el estudiantado y el rendimiento obtenido por los grupos sobre la tarea académica; se destacan tres tipos de discurso socioemocional que parecen tener una incidencia positiva sobre el rendimiento de los grupos: expresiones de ánimo hacia los compañeros, manifestación de emociones y el uso de vocativos inclusivos.

Por su parte, el estudio de Colomina y Remesal (2015) pone de relieve el carácter dinámico y evolutivo del discurso socioemocional de los grupos. Los hallazgos de esta investigación resaltan que, en etapas tempranas de la colaboración, el estudiantado emplea con frecuencia mecanismos discursivos para el fortalecimiento de la cohesión grupal y el sentimiento de competencia del estudiantado, sin embargo, el uso de tales dispositivos tiende a disminuir con el paso del tiempo.

Por otra parte, en el estudio realizado por Järvenoja et al. (en prensa) se diferenciaron tres tipos de regulación emocional en el aprendizaje colaborativo: regulación individual de emociones propias, regulación emocional inducida de un sujeto participante a otro y regulación conjunta de la motivación grupal. Los resultados mostraron que la regulación emocional inducida aparece con mayor frecuencia dentro de los grupos y tiene efectos más positivos sobre el aprendizaje del estudiantado, en contraste con los otros dos tipos de regulación. Además, se concluyó que los grupos con mayor éxito en la tarea académica son aquellos en donde el estudiantado se anima y apoya mutuamente ante circunstancias difíciles, sentimientos negativos o situaciones de estrés.

Pese a las particularidades que presentan los trabajos anteriores, el conjunto de resultados supone un avance significativo en el tema, ya que coinciden al resaltar la importancia que el contexto socioemocional tiene en el aprendizaje colaborativo; no obstante, presentan algunas limitaciones. En primer lugar, estos trabajos establecen una relación directa entre el discurso socioemocional utilizado por el estudiantado con las calificaciones finales obtenidas por 
http://doi.org/10.15359/ree.24-2.4

ROR: https://ror.org/01t466c14 Universidad Nacional, Costa Rica

http://www.una.ac.cr/educare

educare@una.cr

los grupos sobre la tarea académica, y dejan de lado la dimensión sociocognitiva en la que tienen lugar los procesos de revisión y coconstrucción de ideas. En segundo lugar, existe poca evidencia sobre cómo el discurso socioemocional incide en la actividad cognitiva de las personas participantes y qué tipos de discurso se asocian con procesos profundos de construcción compartida del conocimiento. Para profundizar en dichos aspectos, en este trabajo se delimitan tres objetivos específicos:

a) Identificar los tipos de discurso socioemocional que el estudiantado utiliza durante la elaboración tareas colaborativas en línea.

b) Explorar la relación entre el discurso socioemocional utilizado por los grupos de estudiantes y los procesos de construcción compartida del conocimiento.

c) Identificar los tipos de discurso socioemocional que pueden asociarse a un mayor o menor avance y calidad del conocimiento de los grupos.

\section{Metodología}

La estrategia metodológica que se utiliza en esta investigación corresponde a un estudio cualitativo de casos múltiples (Flick, 2002; Stake, 1994; Yin, 2006) en el que se exploran, en profundidad, procesos colaborativos en línea desarrollados por tres pequeños grupos universitarios de estudiantes. En concreto, se eligieron tres casos con la finalidad de comparar las formas de interacción socioemocional que surgen en los grupos y su relación con los procesos de construcción compartida del conocimiento.

\section{Situaciones de observación y participantes}

La investigación se llevó a cabo con estudiantes de licenciatura de la Universidad Autónoma de Baja California, México. Específicamente, los datos corresponden al trabajo desarrollado en el marco de una asignatura obligatoria en la que se abordan contenidos relacionados con la investigación educativa. En detalle, se conformaron tres grupos de trabajo (cada grupo conformado, al azar, por cinco estudiantes, en su mayoría mujeres de entre 19 a 21 años) para la realización de una tarea colaborativa y compleja que consistía en seleccionar y plantear un problema de investigación educativa.

Al inicio de la actividad, en una clase presencial, el profesor explicó las características generales de la tarea y dio instrucciones al estudiantado para que colaborara en un entorno tecnológico de comunicación asíncrona. 
Como parte del producto final se pidió a los grupos que elaboraran un informe escrito respondiendo a las siguientes preguntas directrices: ¿Cuál es el problema de investigación por abordarse? ¿Cómo surge? ¿Por qué surge? ¿Dónde surge? ¿Qué personas están implicadas? ¿Cuáles son sus características? ¿Qué objetivos se plantean para la investigación?

El plazo establecido para la realización de la tarea fue de cinco semanas. Las actividades se desarrollaron en la plataforma educativa“Blackboard", a través de un foro de comunicación asíncrona. Todas las personas participantes contaban con experiencia previa en el uso de la plataforma.

Para acceder a los datos se solicitó, de manera escrita, el consentimiento de cada estudiante. En dicha carta tambien se estableció el código de ética, por parte del equipo de investigación, en cuanto al manejo de los datos y el anonimato de los nombres de participantes. Además, en común acuerdo con el profesor, se tomó la desición de recoger la información al finalizar la tarea, con el propósito de no interferir en el desarrollo natural de las actividades de los grupos de estudiantes.

\section{Análisis de datos}

El corpus de datos analizado son las contribuciones efectuadas por el estudiantado dentro de los foros de comunicación asíncrona, en este sentido, la estrategia utilizada para la introspección de los datos corresponde a la técnica de análisis de contenido.

Según Piñuel (2002), el análisis de contenido es una técnica de carácter cualitativo que permite explorar los procesos comunicativos de manera rigurosa y sistemática. De manera particular, en el caso de las interacciones electrónicas, las unidades de análisis objeto de exploración son los mensajes escritos.

Con base en lo anterior, y teniendo como referencia la propuesta de Coll et al. (2010), el procedimiento de análisis para este trabajo consistió, en un primer momento, en la identificación de cadenas sociocognitivas (Cs) dentro de los foros de trabajo grupal.

Una Cs se define operacionalmente como el conjunto de contribuciones realizadas por diferentes integrantes del grupo que tienen una conectividad conversacional, donde el turno que abre la discusión sobre un eje temático es el punto de arranque de la Cs y el turno que concluye dicho eje y que finaliza dicha conectividad es el punto de cierre. Para identificar las Cs en las contribuciones del estudiantado, se definió un conjunto de criterios de conectividad conversacional que permite establecer tres tipos diferentes de conectividad conversacional: a) conectividad por adyacencia temática, b) conectividad por alusión y c) conectividad por continuidad implícita. La descripción de tales categorías se presenta en la Tabla 1. 
http://doi.org/10.15359/ree.24-2.4

ROR: https://ror.org/01t466c14 Universidad Nacional, Costa Rica

http://www.una.ac.cr/educare

educare@una.cr

Tabla 1: Criterios para la configuración de cadenas sociocognitivas (Cs)

\begin{tabular}{ll}
\hline \multicolumn{1}{c}{ Categoría } & \multicolumn{1}{c}{ Descripción } \\
\hline Conectividad por adyacencia temática & $\begin{array}{l}\text { Continuidad de la conversación entre dos fragmentos de mensajes } \\
\text { que surgen de manera continua en el tiempo y que remiten a un } \\
\text { mismo tema. }\end{array}$ \\
Conectividad por alusión & $\begin{array}{l}\text { Continuidad de la conversación en el que las personas participantes } \\
\text { recurren a la realización de alusiones (a un compañero o documento } \\
\text { adjunto) para retomar la continuidad de la conversación, ya que los } \\
\text { mensajes realizados en torno a una misma línea temática surgen de } \\
\text { manera distanciada en el tiempo. } \\
\text { Réplica implícita a una línea conversacional que se ha planteado con } \\
\text { Conectividad por continuidad implícita } \\
\text { anterioridad, en donde se retoma o finaliza la discusión sobre un } \\
\text { tema en específico. }\end{array}$ \\
\hline
\end{tabular}

Nota: Elaboración propia.

Después, tomando como base las distintas fases teóricas de los procesos de construcción compartida del conocimiento propuestas en otros trabajos (Engel y Onrubia, 2010; Tirado et al., 2011), las Cs inicialmente identificadas en las contribuciones del estudiantado se agruparon en cuatro categorías que se corresponden con dichas fases; la naturaleza de cada categoría se describe en la Tabla 2.

Tabla 2: Clasificación de cadenas sociocognitivas (Cs) identificadas por fases de construcción compartida del conocimiento

\begin{tabular}{lrl}
\hline Categorías/fases del conocimiento & Código & \multicolumn{1}{c}{ Descripción } \\
\hline Fase 1. Organización de la tarea & F_Or & $\begin{array}{l}\text { Cs en donde los miembros del grupo revisan las instrucciones de } \\
\text { la tarea, fijan metas y construyen planes de acción. } \\
\text { Fase 2. Diagnóstico sobre el tema }\end{array}$ \\
Fase 3. Construcción de significados donde los miembros de un grupo efectúan la primera \\
Fase 4. Síntesis y acuerdos finales & F_Cs & $\begin{array}{l}\text { Cs en donde los estudiantes aportan significados para la } \\
\text { aclarando dudas generales sobre el tema } \\
\text { elaboración del informe y discuten sobre el contenido de las } \\
\text { propuestas }\end{array}$ \\
\hline
\end{tabular}

Nota: Elaboración propia. 
http://doi.org/10.15359/ree.24-2.4

ROR: https://ror.org/01t466c14 Universidad Nacional, Costa Rica

Una vez que las Cs se agruparon por fases del conocimiento, se desarrolló un esquema de codificación para analizar el discurso socioemocional utilizado por el estudiantado dentro de cada fase. Como se muestra en la Tabla 3, se propusieron 15 códigos asociados con las categorías de cohesión de grupo, expectativas, valoraciones positivas y componentes afectivos. Vale la pena destacar que los códigos propuestos forman parte de un proceso recursivo en el que dos especialistas del equipo de investigación en el campo de la psicología de la educación discutieron el refinamiento de los códigos hasta llegar a un consenso.

Tabla 3: Sistema de códigos para el análisis del discurso socioemocional en las Cs

\begin{tabular}{|c|c|c|}
\hline Categoría & Código & Descripción \\
\hline \multirow{2}{*}{$\begin{array}{l}\text { Cohesión del } \\
\text { grupo }\end{array}$} & UM_Co1 & Emplean expresiones de cordialidad social \\
\hline & UM_Co2 & $\begin{array}{l}\text { Emplean pronombres inclusivos que transmiten que el grupo funciona como equipo } \\
\text { y no como agentes individuales }\end{array}$ \\
\hline \multirow{2}{*}{ Expectativas } & UM_Ex1 & $\begin{array}{l}\text { Formulan expresiones de autocompetencia sobre la participación individual y el } \\
\text { proceso colaborativo }\end{array}$ \\
\hline & UM_Ex2 & $\begin{array}{l}\text { Formulan expresiones de autocompetencia sobre el desempeño grupal ante la tarea } \\
\text { o elaboración de los productos }\end{array}$ \\
\hline \multirow{3}{*}{$\begin{array}{l}\text { Valoraciones } \\
\text { positivas }\end{array}$} & UM_Vr1 & $\begin{array}{l}\text { Realizan estimaciones sobre las exigencias y el esfuerzo que implica la realización } \\
\text { grupal de la tarea }\end{array}$ \\
\hline & UM_Vr2 & Estiman positivamente el progreso de la tarea y producto final elaborado grupalmente \\
\hline & UM_Vr3 & Estiman positivamente el esfuerzo realizado por sus pares y el grupo \\
\hline \multirow{8}{*}{$\begin{array}{l}\text { Componentes } \\
\text { afectivos }\end{array}$} & UM_Em1 & Comparten emociones vinculadas con experiencias previas a la realización de la tarea \\
\hline & UM_Em2 & $\begin{array}{l}\text { Expresan emociones o estados de ánimo relacionados con la tarea actual o la } \\
\text { asignatura }\end{array}$ \\
\hline & UM_Em3 & $\begin{array}{l}\text { Comparten emociones sobre sucesos personales (no relacionados con la asignatura, } \\
\text { tarea, o grupo) }\end{array}$ \\
\hline & UM_Em4 & Recurren al humor mientras tratan asuntos relacionados con la tarea \\
\hline & UM_Em5 & Palabras de apoyo hacia sus pares para la vivacidad de la tarea \\
\hline & UM_Em6 & Expresan buenos deseos e interés por sus pares \\
\hline & UM_Em7 & Comparten experiencias o emociones relacionadas con el funcionamiento del grupo \\
\hline & UM_Em8 & $\begin{array}{l}\text { Controlan estados motivacionales negativos o regulan emociones negativas que } \\
\text { surgen en/entre miembros del grupo }\end{array}$ \\
\hline
\end{tabular}

Nota: Elaboración propia. 
http://doi.org/10.15359/ree.24-2.4

ROR: https://ror.org/01t466c14 Universidad Nacional, Costa Rica

http://www.una.ac.cr/educare

educare@una.cr

\section{Resultados}

\section{Identificación de Cs y su agrupación por fases de construcción compartida del conocimiento}

En la Tabla 4 se muestran las frecuencias y porcentajes correspondientes a las Cs identificadas en todos los grupos en cada fase; se distinguen los valores grupales superiores al $40 \%$ (celdas sombreadas) de los valores inferiores a dicho porcentaje (celdas sin sombrear). A nivel global, según se observa en las últimas dos columnas de la Tabla 4, se registró un total de 57 Cs; los porcentajes más altos se ubican en los grupos A (38.60 \%) y B (36.84 \%). En detalle, y haciendo una lectura por cada fase, el grupo A se distingue por la alta proporción de Cs identificadas en dos fases: las referidas a la organización de la tarea (F_Or con $43.75 \%$ ) y las de construcción de significados (F_Cs con $42.86 \%$ ); en cambio, el grupo B solo destaca con las Cs desarrolladas en la fase de diagnóstico sobre el tema (F_Dt con $42.11 \%$ ) y el grupo C lo hace con las Cs dirigidas a la fase síntesis y acuerdos finales (F_Sf con 50 \%).

Tabla 4: Identificación y agrupación de Cs por fases de construcción compartida del conocimiento

\begin{tabular}{ccccccccccc}
\hline Grupos & \multicolumn{2}{c}{$\begin{array}{c}\text { Fase 1 } \\
\text { F_Or }\end{array}$} & \multicolumn{2}{c}{$\begin{array}{c}\text { Fase 2 } \\
\text { F_Dt }\end{array}$} & \multicolumn{2}{c}{$\begin{array}{c}\text { Fase 3 } \\
\text { F_Cs }\end{array}$} & \multicolumn{2}{c}{$\begin{array}{c}\text { Fase 4 } \\
\text { F_Sf }\end{array}$} & \multicolumn{2}{c}{ Total } \\
& $f$ & $\%$ & $f$ & $\%$ & $f$ & $\%$ & $f$ & $\%$ & $f$ & $\%$ \\
\hline A & 7 & 43.75 & 7 & 36.84 & 6 & 42.86 & 2 & 25.00 & 22 & 38.60 \\
B & 6 & 37.50 & 8 & 42.11 & 5 & 35.71 & 2 & 25.00 & 21 & 36.84 \\
C & 3 & 18.75 & 4 & 21.05 & 3 & 21.43 & 4 & 50.00 & 14 & 24.56 \\
\hline Total & 16 & 28.07 & 19 & 33.33 & 14 & 24.56 & 8 & 14.04 & 57 & 100.00 \\
\hline
\end{tabular}

Nota: Elaboración propia.

\section{Discurso socioemocional, tipología y diversidad en los grupos}

Dentro de las Cs se constató la presencia de distintos mecanismos discursivos utilizados por el estudiantado para el fortalecimiento del contexto socio-emocional. De acuerdo con la última fila de la Tabla 5, a nivel global, llama la atención la alta proporción de elementos discursivos dirigidos al fortalecimiento de la cohesión de grupo (51.41\% de elementos codificados) y, en menor medida, de las manifestaciones de afectos entre las personas participantes (31.46 \%).

En concreto, el grupo A se destaca por la diversidad de mecanismos discursivos identificados en las Cs, ya que obtiene porcentajes superiores al $40 \%$ en las cuatro categorías contempladas; el dato anterior coincide con la lectura global, ya que de los 391 elementos codificados, el 48,59\% corresponde a este grupo. En menor medida, aunque el grupo B también despliega diversos elementos discursivos, estos se centran mucho más en el fortalecimiento de la cohesión de grupo (41.29 \%) y en la realización de valoraciones positivas sobre el proceso 
http://doi.org/10.15359/ree.24-2.4

ROR: https://ror.org/01t466c14 Universidad Nacional, Costa Rica http://www.una.ac.cr/educare educare@una.cr

colaborativo (44.64\%); al hacer la lectura global, este grupo obtiene poco más del $38 \%$ del total de los elementos codificados. Finalmente, el grupo $C$ se distingue, contrariamente a los otros dos grupos, por el escaso despliegue de recursos discursivos socioemocionales, ya que obtuvo porcentajes por debajo del $16 \%$; de hecho, en este grupo no se identificaron elementos socioemocionales relacionados con la formulación de expectativas.

Tabla 5: Elementos socioemocionales regulados a través del discurso del estudiantado

\begin{tabular}{ccccccccccc}
\hline Grupos & \multicolumn{2}{c}{ Cohesión } & \multicolumn{2}{c}{ Expectativas } & \multicolumn{2}{c}{ Valoraciones } & \multicolumn{2}{c}{ Afectos } & \multicolumn{2}{c}{ Total } \\
\hline & $f$ & $\%$ & $f$ & $\%$ & $f$ & $\%$ & $f$ & $\%$ & $f$ & $\%$ \\
\hline A & 86 & 42.79 & 9 & 81.82 & 25 & 44.64 & 70 & 56.91 & 190 & 48.59 \\
B & 83 & 41.29 & 2 & 18.18 & 25 & 44.64 & 40 & 32.52 & 150 & 38.36 \\
C & 32 & 15.92 & 0 & 0 & 6 & 10.71 & 13 & 10.57 & 51 & 13.04 \\
\hline Total & 201 & $51.41 \%$ & 11 & $2.81 \%$ & 56 & $14.32 \%$ & 123 & $31.46 \%$ & 391 & 100.00 \\
\hline
\end{tabular}

Nota: Elaboración propia.

La Tabla 6 muestra la frecuencia y diversidad con que aparecieron los diversos tipos de discurso socioemocional identificados en las Cs. A nivel global, de los 15 tipos posibles, los que surgieron con mayor frecuencia son los relacionados con la categoría de cohesión de grupo; en efecto, como se aprecia en la última columna de la tabla, el uso de expresiones de cordialidad social (UM_Co1 con 111 elementos codificados) y el uso de pronombres inclusivos para dirigirse al grupo (UM_Co2 con 90 elementos codificados) son los más presentes. En cambio, dentro de la categoría de componentes afectivos prevalecieron tres tipos de discurso: manifestaciones sobre estados de ánimo o emociones vinculadas con la tarea (UM_Em2 con 38 elementos codificados), expresiones de apoyo mutuo entre las personas participantes (UM_Em5 con 28 elementos codificados) y expresiones de buenos deseos e interés por sus pares (UM_Em6 con 21 elementos codificados). En relación con la categoría de valoraciones, sobresalieron más las estimaciones positivas sobre el esfuerzo realizado por las personas participantes/desempeño del grupo (UM_Vr3 con 28 elementos codificados) y aquellas en torno al progreso de la tarea (UM_Vr2 con 23 elementos codificados). Finalmente, los dos tipos de discurso relacionados con la categoría de expectativas, sobre la autocompetencia para el trabajo individual/colaborativo (UM_Ex1) y sobre la tarea académica (UM_Ex2), son elementos discursivos que aparecieron con baja frecuencia dentro de los grupos (4 y 7 elementos identificados, respectivamente).

Al hacer una lectura de las frecuencias en cada grupo, de acuerdo con la Tabla 6 en la que se distinguen los valores iguales o superiores a 10 (celdas sombreadas) de los valores inferiores a dicho número (celdas sin sombrear), los grupos A y B mostraron una mayor densidad y variedad de recursos discursivos socioemocionales durante la realización de la tarea. En detalle, en el 
http://doi.org/10.15359/ree.24-2.4

ROR: https://ror.org/01t466c14 Universidad Nacional, Costa Rica

http://www.una.ac.cr/educare

educare@una.cr

grupo A se desplegaron con mayor frecuencia siete tipos de recursos discursivos: expresiones de cordialidad social (UM_Co1), uso de pronombres inclusivos (UM_Co2), estimaciones positivas en torno al progreso de la tarea (UM_Vr2) y al esfuerzo de las personas participantes/desempeño del grupo (UM_Vr3), manifestaciones sobre estados de ánimo o emociones vinculadas con la tarea (UM_Em2), de apoyo mutuo entre las personas participantes (UM_Em5) y buenos deseos e interés por sus pares (UM_Em6). En menor medida, el estudiantado del grupo B utilizó, de manera recurrente, cinco tipos de discurso socioemocional: UM_Co1, UM_Co2, Um_Vr2, Um_Vr3 y UM_Em5. A diferencia de los grupos A y B, en el grupo $C$ el discurso socioemocional se redujo prácticamente a los recursos relacionados con la cohesión del grupo (UM_Co1 y UM_Co2).

Tabla 6: Discurso socioemocional; tipología y diversidad en los grupos

\begin{tabular}{lccccc}
\hline \multicolumn{1}{c}{ Categorías } & Tipos & Grupo A & Grupo B & Grupo C & Total \\
\hline \multirow{2}{*}{ Cohesión del grupo } & UM_Co1 & 42 & 47 & 22 & 111 \\
& UM_Co2 & 44 & 36 & 10 & 90 \\
\hline \multirow{2}{*}{ Expectativas } & UM_Ex1 & 4 & 0 & 0 & 4 \\
& UM_Ex2 & 5 & 2 & 0 & 7 \\
\hline \multirow{3}{*}{ Valoraciones positivas } & UM_Vr1 & 1 & 4 & 0 & 5 \\
& UM_Vr2 & 10 & 11 & 2 & 23 \\
& UM_Vr3 & 14 & 10 & 4 & 28 \\
\hline \multirow{5}{*}{ Componentes afectivos } & UM_Em1 & 6 & 3 & 0 & 9 \\
& UM_Em2 & 26 & 7 & 5 & 38 \\
& UM_Em3 & 1 & 0 & 0 & 1 \\
& UM_Em4 & 5 & 3 & 0 & 8 \\
& UM_Em5 & 14 & 14 & 0 & 28 \\
& UM_Em6 & 13 & 4 & 4 & 21 \\
& UM_Em7 & 2 & 4 & 3 & 9 \\
& UM_Em8 & 3 & 5 & 1 & 9 \\
\hline & TOTAL & $190 / 15^{*}$ & $150 / 13^{*}$ & $51 / 8^{*}$ & $391 / 15^{*}$ \\
\hline
\end{tabular}

* Los asteriscos representan el número de los distintos tipos de recursos discursivos utilizados por los grupos, de los 15 tipos posibles.

Nota: Elaboración propia. 
http://doi.org/10.15359/ree.24-2.4

ROR: https://ror.org/01t466c14 Universidad Nacional, Costa Rica

\section{Discurso socioemocional y fases de construcción compartida del conocimiento}

Las siguientes figuras (Figura 1, Figura 2 y Figura 3) representan la presencia y estabilidad de los recursos discursivos socioemocionales desplegados por los diferentes grupos en las distintas fases de construcción del conocimiento.

En el caso del grupo A, de acuerdo con la Figura 1, se observa una presencia alta y estable del discurso socioemocional utilizado por el estudiantado especialmente en dos categorías. En detalle, de las cuatro categorías asociadas con este tipo de discurso, la cohesión grupal se mantiene de manera positiva en las cuatro fases del conocimiento, mientras que los recursos asociados con la comunicación afectiva adquieren mayor relevancia tanto en la fase de construcción de significados (Fase 3) como en la fase de toma de síntesis/acuerdos finales (Fase 4). En cambio, las valoraciones positivas aparecen con mayor frecuencia en la fase de diagnóstico sobre el tema (Fase 2) y la Fase 3, sin embargo, bajan en la Fase 4. Sobre los recursos asociados con las expectativas, se observa que son elementos discursivos que surgen puntualmente durante la fase de organización de la tarea (Fase 1) y en la Fase 4.

Figura 1: Representación del discurso socioemocional del grupo A por fases del conocimiento

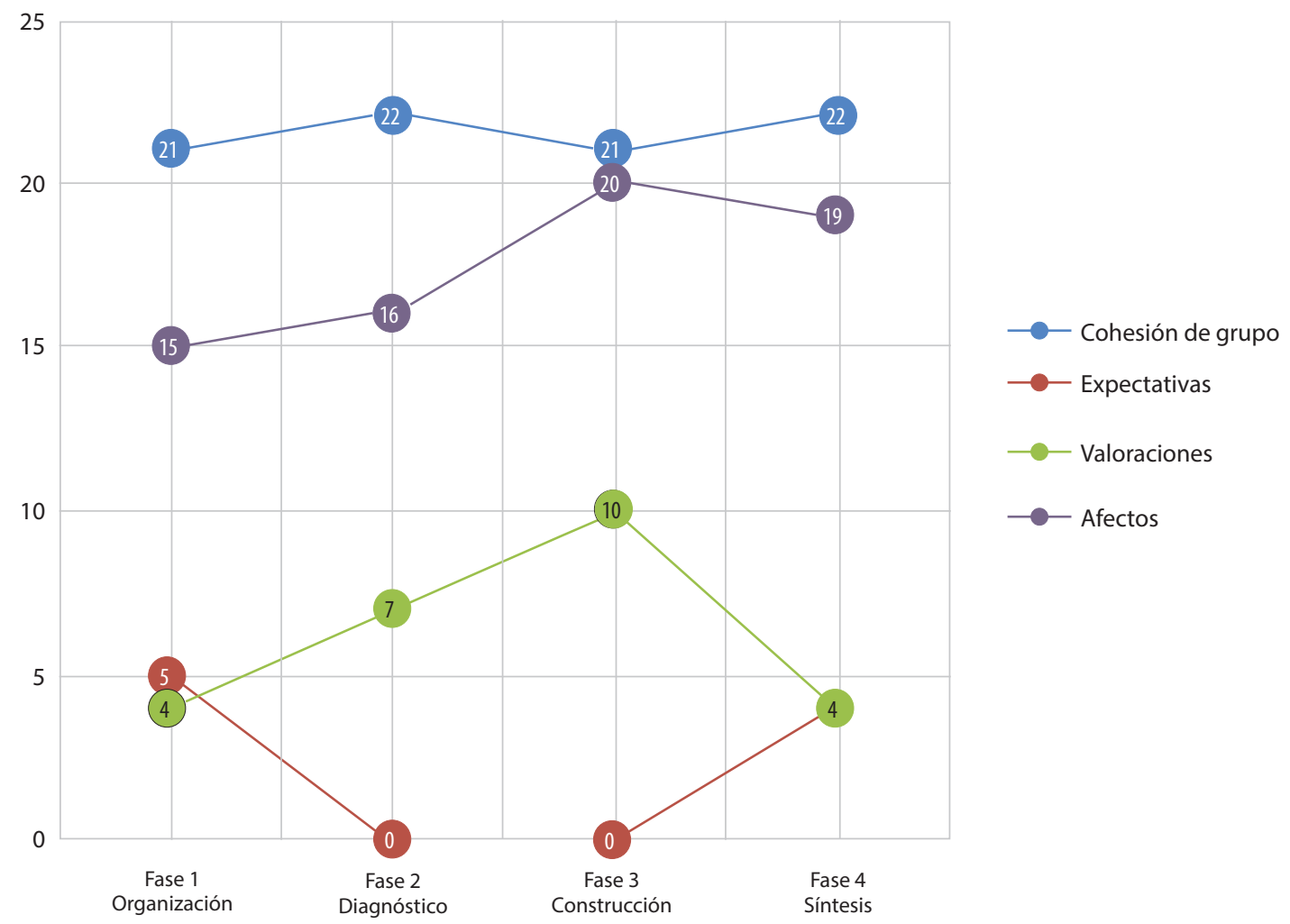

Nota: Elaboración propia. 
http://doi.org/10.15359/ree.24-2.4

ROR: https://ror.org/01t466c14 Universidad Nacional, Costa Rica

http://www.una.ac.cr/educare

educare@una.cr

Por lo que respecta al grupo B, de acuerdo con la Figura 2, se constata que el discurso socioemocional desplegado por el estudiantado se incrementa paulatinamente especialmente en una categoría. En efecto, los recursos discursivos asociados a la cohesión de grupo aumentaron entre cada fase hasta adquirir su máximo valor en la Fase 4. Sobre los recursos afectivos se observa que estos se mantienen más o menos estables y alcanzan su máximo valor en la Fase 3. Aunque los recursos asociados con las valoraciones positivas aumentan gradualmente entre la Fase 1 y la Fase 3, estos disminuyen considerablemente en la Fase 4. Finalmente, la formulación de expectativas solo se observó, mínimamente, dentro de la Fase 1.

Figura 2: Representación del discurso socioemocional del grupo B por fases del conocimiento.

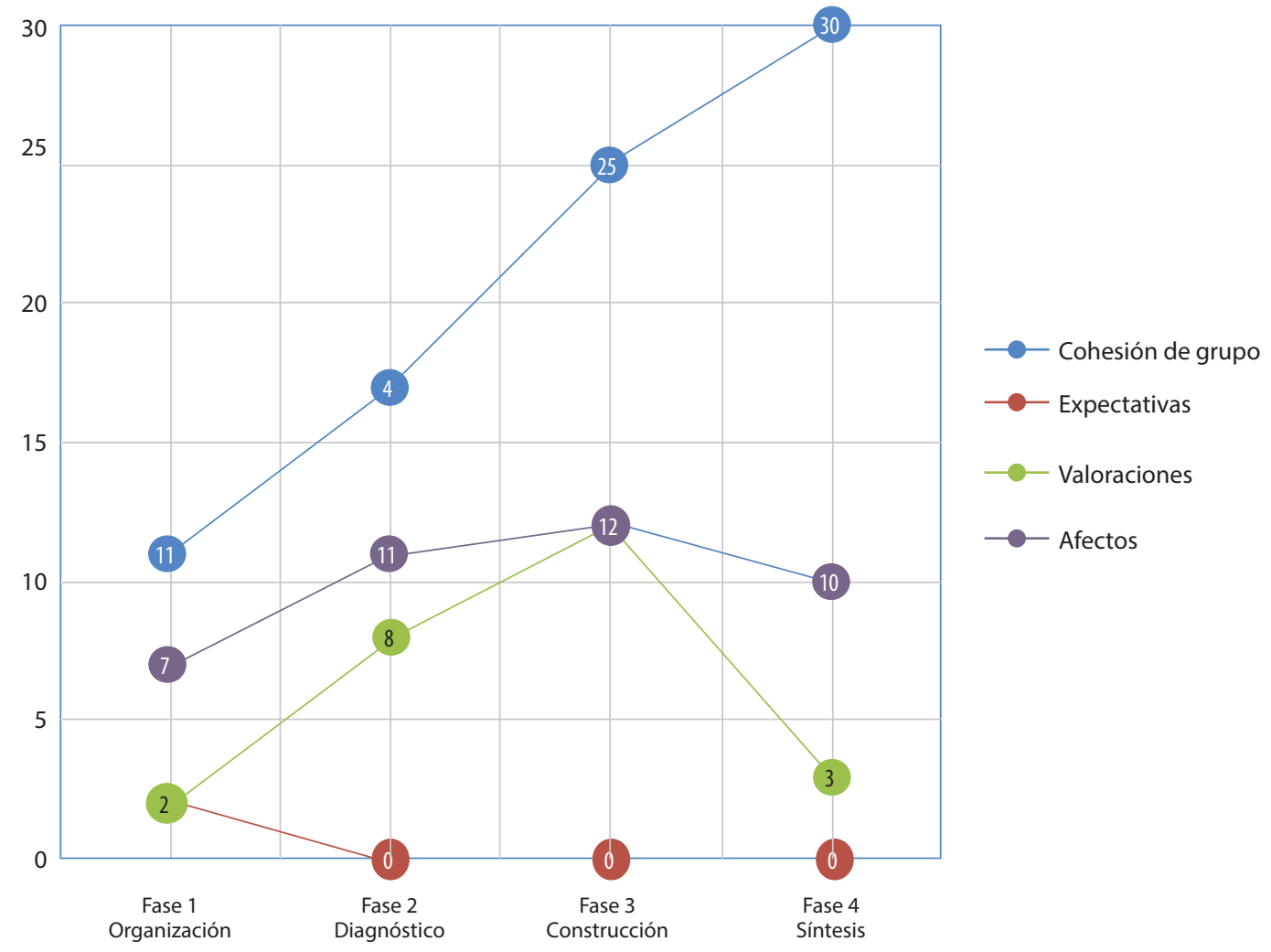

Nota: Elaboración propia.

Finalmente, según los datos que muestra la Figura 3 y contrariamente a lo observado en los grupos anteriores, el grupo C se caracteriza por el declive progresivo en el discurso socioemocional del estudiantado. En detalle, aunque la cohesión de grupo y la comunicación afectiva aparecen en la Fase 1 con valores altos, los recursos asociados con dichas categorías descienden fuertemente en 
http://doi.org/10.15359/ree.24-2.4

ROR: https://ror.org/01t466c14 Universidad Nacional, Costa Rica

las fases posteriores. Algo similar ocurre con los recursos asociados con las valoraciones positivas que disminuyen a tal punto que no están presentes en la última fase. Cabe destacar que en este grupo no se observaron formulaciones de expectativas en ninguna de las fases analizadas.

Figura 3: Representación del discurso socioemocional del grupo $C$ por fases del conocimiento.

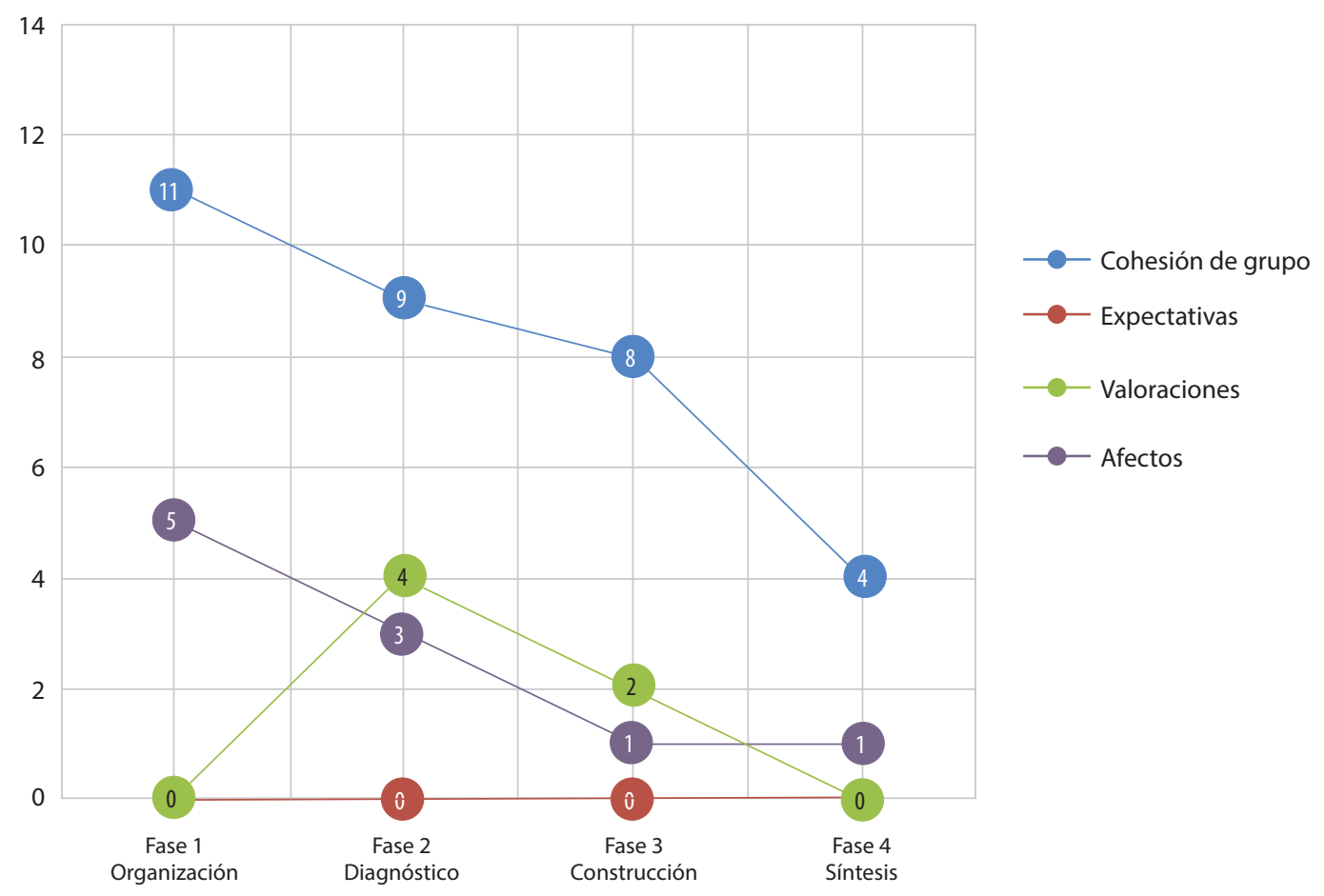

Nota: Elaboración propia.

\section{Discusión}

El propósito de este trabajo consistió en explorar el discurso socioemocional que distintos grupos de estudiantes utilizan durante la elaboración de tareas colaborativas en línea, así como el efecto que determinados tipos de discurso socioemocional tienen en los procesos de construcción compartida del conocimiento. Para ello, primero, se identificaron Cs en la interacción global de los grupos y, después, estas se agruparon en cuatro fases: organización de la tarea, diagnóstico del tema, construcción de significados y síntesis/acuerdos finales.

En términos globales, se observó que los mecanismos discursivos más utilizados por el estudiantado son aquellos dirigidos a favorecer la cohesión de grupo y los relacionados con las manifestaciones de afectos. También se constató que los grupos con un alto 
http://doi.org/10.15359/ree.24-2.4

ROR: https://ror.org/01t466c14 Universidad Nacional, Costa Rica

http://www.una.ac.cr/educare

educare@una.cr

número de Cs son los que destacan por la diversidad de mecanismos discursivos desplegados y lo hacen, especialmente, a través de siete tipos diferentes de discurso socioemocional: expresiones de cordialidad social, el uso de pronombres inclusivos, manifestaciones sobre estados de ánimo, emociones vinculadas con la tarea, expresiones de apoyo mutuo entre las personas participantes y de buenos deseos, las estimaciones positivas sobre el esfuerzo realizado por participantes, y valoraciones positivas sobre el progreso de la tarea.

Otro resultado interesante remite al hecho de que no solo la densidad y frecuencia del discurso socioemocional es importante, sino que también se requiere de uso variado y continuo de las estrategias discursivas a través de las fases de construcción del conocimiento. En detalle, tal como se observó en este trabajo, los grupos (grupo A y grupo B) que sostuvieron un discurso socio-emocional denso, heterogéneo y estable desarrollaron un mayor número de cadenas sociocognitivas centradas en las fases de organización de la tarea, diagnóstico inicial del tema y construcción de significados, a diferencia del grupo $\mathrm{C}$ que se caracterizó por el declive progresivo del discurso socioemocional desplegado. Estos hallazgos amplían los resultados de trabajos previos (Colomina y Remesal, 2015; Kwon et al., 2014; Järvenojä et al., en prensa; Zheng y Huang, 2016), ya que no solo coinciden en la importancia que tiene la frecuencia con que emerge el discurso socioemocional sobre el rendimiento del estudiantado en la tarea académica, sino que, además, conceden importancia tanto a la diversidad como a la estabilidad del discurso socioemocional para incidir de manera positiva en el rendimiento de los grupos.

Otros hallazgos obtenidos a partir del estudio sugieren que el discurso socioemocional heterogéneo es fundamental, sobre todo, durante las fases iniciales del proceso de construcción del conocimiento. Así, en la fase de organización de la tarea, los grupos (a excepción del grupo C) mostraron una combinación de elementos discursivos relacionados con el fortalecimiento de la cohesión de grupo, las relaciones afectivas, las valoraciones satisfactorias y las expectativas positivas sobre la tarea; la combinación de tales elementos provocó un mejor desarrollo de las fases subsiguientes. Estos hallazgos son similares a los expuestos por Malmberg et al. (2017), quienes mostraron que la instauración temprana de un clima socioemocional determina el funcionamiento posterior de los grupos y ayuda a que el estudiantado se involucre en discusiones cada vez más ricas y complejas.

En relación con el punto anterior es preciso señalar que, durante la fase de organización de la tarea, la formulación de expectativas es un elemento discursivo que ocurre con baja frecuencia dentro de los grupos; a pesar de ello, se considera que una mínima cantidad de expresiones de autocompetencia sobre la participación y la tarea son indispensables para alcanzar niveles más profundos de construcción del conocimiento. De acuerdo con Colomina y Remesal (2015) y Schoor y Bannert (2012), las expectativas -entendidas como un sentimiento de autocompetencia grupal- influyen en la motivación del estudiantado y determinan 
fuertemente las actuaciones futuras sobre la tarea, por lo que suelen ser es uno de los mejores predictores en los aprendizajes del estudiantado.

Los resultados también destacan el carácter dinámico y evolutivo que tiene el discurso socioemocional utilizado por el estudiantado. Al igual que Colomina y Remesal (2015), se concluye que cuando la cohesión de grupo y el discurso afectivo entre las personas participantes se incrementan a través de las fases del conocimiento, tal como pasó en los grupos A y B, la calidad de la discusión de las personas participantes también va en aumento. Por su parte, un declive en el discurso socioemocional podría estar indicando un deterioro de la actividad cognitiva y, en consecuencia, un menor avance en el conocimiento grupal, como sucedió con el grupo $C$.

Finalmente se destacan algunas limitaciones de este trabajo y líneas abiertas para la realización de próximos estudios. En primer lugar, se reconoce que el número de casos analizados es reducido y se requiere mayor evidencia para dar robustez a las conclusiones, por lo que estas deben tomarse con cierta cautela. En segundo lugar, se considera que la aplicación de pruebas estadísticas de correspondencia hubiera ayudado a identificar patrones de relación entre los distintos tipos de discurso socioemocional; en este sentido, se plantea que futuras investigaciones deben profundizar en las distintas fases de construcción compartida del conocimiento con una doble mirada, es decir, analizar en paralelo tanto el discurso de orden socioemocional como las estrategias discursivas de orden cognitivo que se presentan en cada fase. Otra de las limitaciones de este trabajo tiene que ver con los códigos utilizados para valorar el contexto socioemocional de los grupos, ya que solo remiten a la presencia de discursos positivos, a pesar de que en la interacción entre estudiantes también se observaron discursos socioemocionales negativos (que no fueron codificados) y que probablemente afectaron el desempeño de los grupos; futuras investigaciones deberían considerar la posibilidad de incluir códigos antagónicos (discurso socioemocional positivo Vs. negativo) para valorar el contexto socioemocional desde una mirada más amplia.

\section{Conclusiones}

La solidez de los hallazgos presentados y discutidos han permitido generar las siguientes conclusiones. En primer lugar, se concluye que cuando los grupos de estudiantes despliegan un alto número de Cs durante su interacción en los foros es porque concentran su trabajo, especialmente, en las primeras tres fases de construcción del conocimiento: i) organización de la tarea, ii) diagnóstico sobre el tema y iii) construcción de significados. Además, los grupos con mejor desempeño en los foros asíncronos suelen destacarse por la diversidad de mecanismos discursivos de tipo socioemocional utilizados para apoyar y sostener interacciones productivas sobre la tarea. 
http://doi.org/10.15359/ree.24-2.4

ROR: https://ror.org/01t466c14 Universidad Nacional, Costa Rica

http://www.una.ac.cr/educare

educare@una.cr

En segundo lugar, se concluye que la presencia -o ausencia- del discurso socioemocional incide tanto en la calidad de la construcción del conocimiento como en los procesos colaborativos de los sujetos participantes. En efecto, cuando los grupos sostienen un discurso socioemocional denso, heterogéneo y estable durante todo el desarrollo de la tarea, no solo muestran un mayor número de cadenas sociocognitivas, sino que además se crea un ambiente de trabajo agradable entre participantes, el cual afecta de manera positiva la cohesión del grupo y sienta las bases para un diálogo abierto, flexible y constructivo.

Finalmente, esta investigación pone de relieve que los mecanismos discursivos que más favorecen la calidad tanto del proceso de construcción del conocimiento como del proceso colaborativo deben cumplir, con al menos, dos exigencias: i) deben ser diversos, especialmente, debe considerarse la combinación de elementos discursivos relacionados con el fortalecimiento de la cohesión de grupo, las relaciones afectivas, las valoraciones satisfactorias y las expectativas positivas sobre la tarea; y ii) la combinación de tales elementos es fundamental, sobre todo, en las fases iniciales de los procesos, ya que genera un mejor desarrollo de las fases subsiguientes.

\section{Agradecimientos}

Esta investigación se ha realizado con recursos púbicos otorgados por la Secretaría de Educación Pública de México, a través del programa para el Desarrollo Profesional Docente, para el Tipo Superior (PRODEP).

\section{Referencias}

Bakhtiar, A., Webster, E. A. y Hadwin, A. F. (2018). Regulation and socio-emotional interactions in a positive and a negative group climate. Metacognition Learning, 13(1), 57-90. https://doi. org/10.1007/s11409-017-9178-x

Castellanos Ramírez, J. C. y Onrubia Goñi, J. (2015). La regulación compartida en entornos de aprendizaje colaborativo: Una revisión del estado de la investigación empírica. Education in the knowledge society, 16(3), 57-72. https://doi.org/10.14201/eks20151635772

Castellanos Ramírez, J.C., Onrubia Goñi, J.y Niño Carrasco, S. A. (2016). El aprendizaje colaborativo mediado por ordenador: Avances y desafíos desde una perspectiva psicoeducativa. En C. E. Román, M. Porras, C. A. D. A. Madrigal y P. Medina (Eds.), Las ciencias de la educación en el proceso de formación del profesional (pp. 70-85). REDEM. www.reed-edu.org/wp-content/ uploads/2016/12/libro-REED-1.pdf

Coll, C., de Gispert, I. y Rochera, M. J. (2010). Tópicos y cadenas: Una aproximación al análisis de la construcción conjunta de significados en foros de conversación en línea. Cultura y Educación, 22(4), 439-454. https://doi.org/10.1174/113564010793351885 
http://doi.org/10.15359/ree.24-2.4

ROR: https://ror.org/01t466c14 Universidad Nacional, Costa Rica http://www.una.ac.cr/educare

Colomina, R. y Remesal, A. (2015). Social presence and virtual collaborative learning processes in higher education. Infancia y Aprendizaje, 38(3), 647-680. https://doi.org/10.1080/0210 $\underline{3702.2015 .1054664}$

Dennen, V. P. y Wieland, K. (2007). From interaction to intersubjectivity: Facilitating online group discourse processes. Distance Education, 28(3), 281-297. ttps://doi. org/10.1080/01587910701611328

Dillenbourg, P., Baker, M., Blaye, A. y O'Malley, C. (1996). The evolution of research on collaborative learning. En P. Reiman y H. Spada (Eds.), Learning in humans and machine: Towards an interdisciplinary learning science (pp. 189-211). Elsevier.

Engel, A. y Onrubia, J. (2010). Patrones de organización grupal y fases de construcción del conocimiento en entornos virtuales de aprendizaje colaborativo. Infancia y Aprendizaje, 33(4), 515-528. https://doi.org/10.1174/021037010793139608

Engel, A. y Onrubia, J. (2013). Estrategias discursivas para la construcción colaborativa del conocimiento en entornos virtuales de aprendizaje. Cultura y Educación, 25(1), 77-94. https://doi.org/10.1174/113564013806309082

Flick, U. (2002). Qualitative research - State of the art. Social Science Information, 41(1), 5-24. https://doi.org/10.1177/0539018402041001001

Janssen, J., Erkens, G., Kirschner, P. A. y Kanselaar, G. (2012). Task-related and social regulation during online collaborative learning. Metacognition and Learning, 7(1), 25-43. https://doi. org/10.1007/s11409-010-9061-5

Järvelä, S. y Hadwin, A. F. (2013). New frontiers: Regulating learning in CSCL. Educational Psychologist, 48(1), 25-39. https://doi.org/10.1080/00461520.2012.748006

Järvenoja, H., Järvelä, S. y Malmberg, J. (En prensa). Supporting groups' emotion and motivation regulation during collaborative learning. Learning and Instruction. https://doi. org/10.1016/j.learninstruc.2017.11.004

Jeong, H. y Hmelo-Silver, C. E. (2016). Seven affordances of computer-supported collaborative learning: How to support collaborative learning? How can technologies help? Educational Psychologist, 51(2), 247-265. https://doi.org/10.1080/00461520.2016.1158654

Johnson, C. M. (2016). Rethinking online discourse: Improving learning through discussions in the online classroom. Education and Information Technologies, 21(6), 1483-1507. https:// doi.org/10.1007/s10639-015-9395-3

Johnson, D. W. y Johnson, R. T. (1999). Making cooperative learning work. Theory into Practice, 38(2), 67-73. https://doi.org/10.1080/00405849909543834 
http://doi.org/10.15359/ree.24-2.4

ROR: https://ror.org/01t466c14 Universidad Nacional, Costa Rica

http://www.una.ac.cr/educare

educare@una.cr

Kirschner, P. A.y Erkens, G. (2013). Toward a framework for CSCL research. Educational Psychologist, 48(1), 1-8. https://doi.org/10.1080/00461520.2012.750227

Kirschner, F., Paas, F., Kirschner, P. A. y Janssen, J. (2011). Differential effects of problem-solving demands on individual and collaborative learning outcomes. Learning and Instruction, 21(4), 587-599. https://doi.org/10.1016/j.learninstruc.2011.01.001

Kwon, K., Liu, Y-H. y Johnson, L. P. (2014). Group regulation and social-emotional interactions observed in computer supported collaborative learning: Comparison between good vs. poor collaborators. Computers \& Education, 78, 185-200. https://doi.org/10.1016/j. compedu.2014.06.004

Kyndt, E., Raes, E., Lismont, B., Timmers, F., Cascallar, E. y Dochy, F. (2013). A meta-analysis of the effects of face-to-face cooperative learning. Do recent studies falsify or verify earlier findings? Educational Research Review, 10, 133-149. https://doi.org/10.1016/j. edurev.2013.02.002

Liang, L. L., Ebenezer, J. y Yost, D. S. (2010). Characteristics of pre-service teachers' online discourse: The study of local streams. Journal of Science Education and Technology, 19(1), 69-79. https://doi.org/10.1007/s10956-009-9179-x

Malmberg, J., Järvelä, S. y Järvenoja, H. (2017). Capturing temporal and sequential patterns of self, $\mathrm{CO}$, and socially shared regulation in the context of collaborative learning. Contemporary Educational Psychology, 49, 160-174. https://doi.org/10.1016/j.cedpsych.2017.01.009

Näykki, P., Järvelä, S., Kirschner, P. A. y Järvenoja, H. (2014). Socio-emotional conflict in collaborative learning: A process-oriented case study in a higher education context. International Journal of Educational Research, 68, 1-14. https://doi.org/10.1016/j.ijer.2014.07.001

Retnowati, E., Ayres, P. y Sweller, J. (2017). Can collaborative learning improve the effectiveness of worked examples in learning mathematics? Journal of Educational Psychology, 109(5), 666-679. https://doi.org/10.1037/edu0000167

Rimor, R., Rosen, Y. y Naser, K. (2010). Complexity of social interactions in collaborative learning: The case of online database environment. Interdisciplinary Journal of E-Learning and Learning Objects, 6(1), 355-365. https://doi.org/10.28945/1321

Schoor, C. y Bannert, M. (2012). Exploring regulatory processes during a computer-supported collaborative learning task using process mining. Computers in Human Behavior, 28(4), 1321-1331. https://doi.org/10.1016/j.chb.2012.02.016 
http://doi.org/10.15359/ree.24-2.4

ROR: https://ror.org/01t466c14 Universidad Nacional, Costa Rica

Splichal, J. M., Oshima, J. y Oshima, R. (2018). Socio-emotional regulation in knowledge building mediated by CSCL reflection. En J. Kay y R. Luckin (Eds.), Rethinking learning in the digital age. Making the learning sciences count, 13th International Conference of the Learning Sciences (ICLS) (Vol. 3, pp. 1013-1016). London, UK: International Society of the Learning Sciences. https://repository.isls.org/bitstream/1/545/1/222.pdf

Stake, R. (1994). Case study. En N. K. Denzin e Y. S. Lincoln (Eds.), Handbook of qualitative research (pp. 236-247). Sage.

Tirado R., Hernando Á. y Aguaded, J. I. (2011). Aprendizaje cooperativo on-line a través de foros en un contexto universitario: Un análisis del discurso y de las redes. Estudios sobre educación, 20, 49-71. rabida.uhu.es/dspace/bitstream/.../Aprendizaje cooperativo contexto universitario.pdf?...

Yin, R. K. (2006). Case study methods. En J. L. Green, G. Camilli y P. B. Elmore (Eds.), Handbook of complementary methods in education research (pp. 111-122). Lawrence Erlbaum Associates.

Zhang, H. (2013). Pedagogical challenges of spoken English learning in the Second Life virtual world: A case study. British Journal of Educational Technology, 44(2), 243-254. https://doi. org/10.1111/j.1467-8535.2012.01312.x

Zheng, L. y Huang, R. (2016). The effects of sentiments and co-regulation on group performance in computer supported collaborative learning. The Internet and Higher Education, 28, 5967. https://doi.org/10.1016/j.iheduc.2015.10.001

Zschocke, K., Wosnitza, M. y Bürger, K. (2016). Emotions in group work: Insights from an appraisaloriented perspective. European Journal of Psychology of Education, 31(3), 359-384. https:// doi.org/10.1007/s10212-015-0278-1 